\title{
Optimization of high-order harmonic generation by genetic algorithm and wavelet time-frequency analysis of quantum dipole emission
}

\author{
Xi Chu and Shih-I Chu \\ Department of Chemistry, University of Kansas, Lawrence, Kansas 66045
}

(Received 7 April 2001; published 13 July 2001)

\begin{abstract}
We present an $a b$ initio three-dimensional quantum study of the coherent control of high-order harmonic generation (HHG) processes in intense pulsed laser fields by means of the genetic algorithm optimization of the laser-pulse amplitude and phase. Accurate time-dependent wavefunction and HHG power spectrum are obtained by the time-dependent generalized pseudospectral method and wavelet transform is used to obtain the dynamical phase associated with the dipole-emission time profile. It is shown that "intra-atomic" dynamical phase matching on the sub-optical cycle, attosecond, time scale can be achieved, leading to nearly perfect constructive interference between different returning electronic wave packets and marked improvement in both emission intensity and purity of a given harmonic order.
\end{abstract}

DOI: 10.1103/PhysRevA.64.021403

PACS number(s): 42.50.Hz, 32.80.Qk, 42.65.Ky, 32.80.Wr

The study of coherent control of atomic and molecular processes is a subject of much current interest in science and technology [1]. In particular, temporally shaped ultrashort laser pulses have been successfully used to design welldefined wave packets [2], and to control multiphoton absorption [3] and chemical reactions [4], etc. In the area of the interaction of atoms with intense-laser-pulse, high-order harmonic generation (HHG) of orders as high as 300 has been observed [5,6], with photon energies in excess of $500 \mathrm{eV}$. A novel concept of "intra-atomic" phase matching has been recently introduced, allowing the enhancement of the intensity of a specific high harmonic [7]. It is shown that by carefully tailoring the shape of the temporal profile of the driving laser pulse, one can control the time evolution of the electron response to the intense laser field on a sub-opticalcycle, attosecond time scale [7]. A semiclassical model [8,9] is used to interpret these results in terms of the constructive interference in the frequency domain between different electron trajectories [10], but the effect of atomic structure is not considered.

In this Rapid Communication, we present a fully ab initio quantum treatment of the coherent control and enhancement of high-harmonic emission by means of the genetic algorithm (GA) optimization [11] of the laser-pulse shape and intra-atomic phase matching. We show that by combining the GA search algorithm and accurate quantum solution of the time-dependent Schrödinger equation, an optimal laser-pulse field can be identified, allowing the enhancement of a given high-harmonic intensity by at least one order of magnitude. Moreover, the study provides physical insights regarding the role of the quantum dynamical phase of the dipole-emission time profile for the coherent control of intra-atomic phase matching, taking into account the effect of atomic structure.

We consider the optimization of the HHG intensity of atomic $\mathrm{H}$ driven by intense linearly polarized (LP) ultrashort laser pulsed fields. We first outline the procedure for the numerical solution of the time-dependent Schrödinger equation, $i \partial / \partial t \Psi(t)=H(t) \Psi(t)$, where $H(t)=H_{0}+V(t)$. $H_{0}$ is the unperturbed Hamiltonian and $V(t)$ is the coupling of the electron with the laser pulse: $V(t)=-\operatorname{Re}[\epsilon(t)] z$, where $\epsilon(t)$ is the electric field. The time-dependent Schrödinger equa- tion is solved accurately and efficiently by the timedependent generalized pseudospectral (TDGPS) method recently developed [12]. The radial coordinate is discretized by the generalized pseudospectral technique [13], allowing nonuniform spatial grid spacing: a denser mesh near the origin and a sparser mesh for the outer regime. The time propagation of the wave function is achieved by the second-order split-operator method in the energy representation [12]. As demonstrated in our recent studies of both strong-field HHG processes of atomic $\mathrm{H}[12,14]$, rare-gas atoms [15], and $\mathrm{H}_{2}$ [16], as well as Rydberg-atom high-resolution spectroscopy [17], the TDGPS procedure is capable of providing a highprecision time-dependent wave function and is computationally more efficient than the conventional time-dependent techniques using equal-spacing grid discretization. The unitarity of the wave function is preserved in the TDGPS procedure. The energy $(=-0.5000000000000$ a.u. $)$ and the norm of the field-free ground-state wave function is preserved to at least ten digits of accuracy during the whole time propagation.

Figure 1 shows the HHG power spectrum $|d(\omega)|^{2}$ of atomic $\mathrm{H}$ driven by $800 \mathrm{~nm}$, Gaussian laser pulse with pulse length $10 \mathrm{fs}$ and laser intensity $2 \times 10^{14} \mathrm{~W} / \mathrm{cm}^{2}$, obtained by the Fourier transform of the induced dipole and dipole acceleration [12], respectively. Excellent agreement of the two spectra is obtained from the lowest harmonic to the cutoff, indicating full convergence of the time-dependent wave functions. The power spectrum shows the typical sharp decline of the harmonic intensity for the first few harmonics, then a plateau consisting of a number of harmonics of similar intensity, followed by a cutoff. As seen in Fig. 1, the few harmonic peaks near the cutoff are structureless, while for harmonics in the plateau below the cutoff, they possess multiple-peak fine structures. As shown in our recent timefrequency analysis of the HHG power spectrum of $\mathrm{H}$ [14] and $\mathrm{H}_{2}$ [16], these fine structures carry significant information regarding the $\mathrm{HHG}$ mechanisms in different harmonic regimes.

Figure 2 shows the dipole-emission time profile of a typical harmonic below the cutoff, the 27 th harmonic in this 


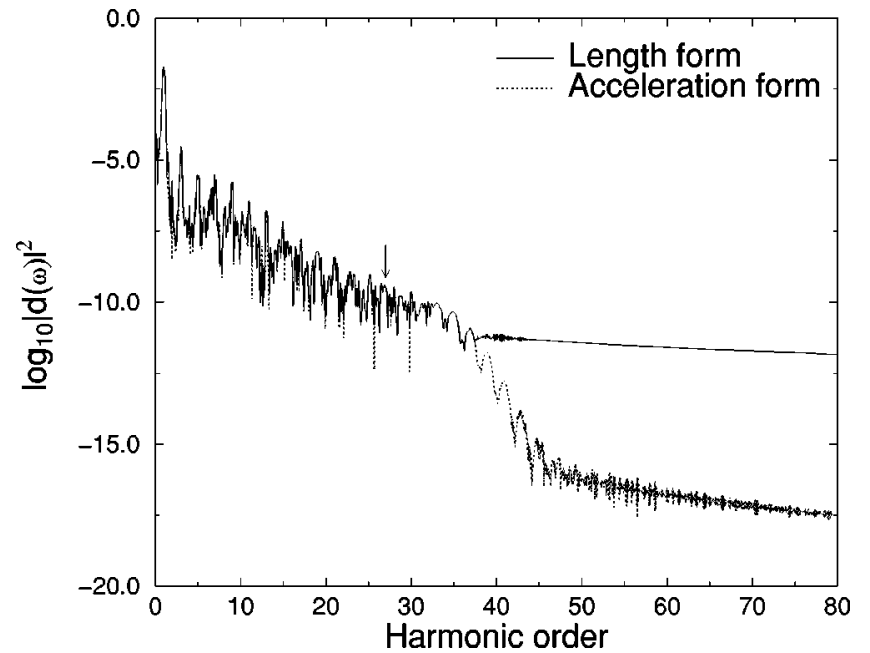

FIG. 1. The HHG power spectrum of a $\mathrm{H}$ atom in a $10 \mathrm{fs}, 800$ $\mathrm{nm}$, Gaussian-pulse-shape laser field with peak intensity 2 $\times 10^{14} \mathrm{~W} / \mathrm{cm}^{2}$. Both the length form (solid line) and acceleration form (dotted line) power spectra are shown for comparison.

case, obtained by the wavelet transform of the induced dipole $d(t)$ (or dipole acceleration) $[14,16,18]$,

$$
A_{w}\left(t_{0}, \omega\right)=\int d(t) w_{t_{0}, \omega}(t) d t \equiv d_{\omega}(t),
$$

where $w_{t_{0}, \omega}(t)=\sqrt{\omega} W\left(\omega\left(t-t_{0}\right)\right)$ is the wavelet kernel. In our study, we choose the Morlet wavelet $W(x)$ $=(1 / \tau) e^{i x} e^{-x^{2} / 2 \tau^{2}}$ as the mother wavelet $[14,18]$. Since the 27 th harmonic peak has a multiplet structure, we present only the results for the two main subpeaks labeled " 1 " and "2." As shown, their dipole time profiles $d_{\omega}(t)$ nearly coincide. This indicates that all the harmonic subpeaks within a given plateau harmonic are produced by the same mecha-

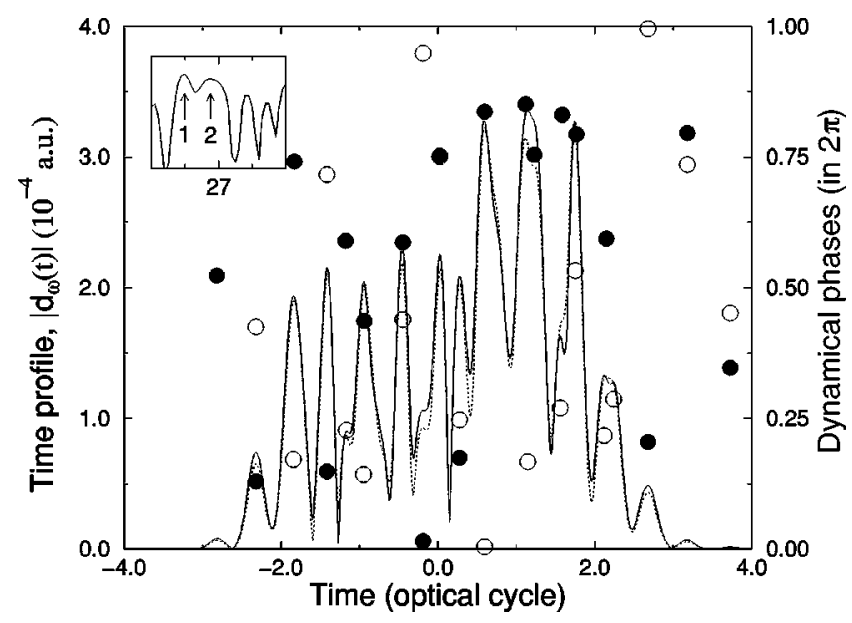

FIG. 2. The dipole-emission time profiles of the subpeaks (labeled " 1 " and " 2 ") of H27: solid line - subpeak " 1 "; dashed line - subpeak "2." The dynamical phases $\theta\left(t_{q}\right)$, corresponding to those "peak" emission times $t_{q}$, are shown as open circles and black dots for the dipole time profiles of the subpeak 1 and 2, respectively. The laser parameters are the same as those in Fig. 1. nism, namely, they are produced by the interference in time of the bremsstrahlung radiations emitted from all the returning electronic wave packets within the incident laser-pulse duration $[14,16]$. Also shown in Fig. 2 are the "dynamical phases" $\theta\left(t_{q}\right)$ of the dipole-emission time profile $d_{\omega}(t)$ (denoted by black dots and open circles for the case of 1 and 2 subpeak, respectively), corresponding to the "peak" emission times $t_{q}$, i.e., those instants where the maxima of $d(t)$ occur: $d_{\omega}\left(t_{q}\right)=\left|d_{\omega}\left(t_{q}\right)\right| e^{-i S\left(t_{q}\right)}$, where $S\left(t_{q}\right)=\omega t_{q}+\theta\left(t_{q}\right)$. These instants may be semiclassically interpreted as the electron-ion recollision times. Figure 2 shows that the dynamical phase varies rather rapidly with time and spans the whole range of values, between 0 and $2 \pi$, leading to both constructive and destructive interference of the dipole emission in different times. To enhance the light emission intensity for any given harmonic, it is thus essential to control these dynamical phases on a sub-optical-cycle time scale to achieve the optimal intra-atomic (dynamical) phase matching. We show below that this can be accomplished by the genetic algorithm optimization procedure.

Genetic algorithm has become a popular approach for optimization [11]. It simulates the natural selection, where stronger individuals are the likely survivors in a challenging environment. The fundamental unit of genetic information in a living cell is the gene. It determines or affects a single character or visible property, for example, eye color. It comprises a segment of DNA, which is packed into structures called chromosomes. The genetic information in the chromosomes of the children are altered compared to their parents by genetic operators such as crossover and random mutation. Crossover exchanges corresponding genetic material from the chromosomes of the parents, allowing beneficial genes on different parents to be combined in their children. Random mutation provides background variation and occasionally introduces beneficial material into a species' chromosomes. GA uses a direct analogy of such natural evolution. GA presumes that the potential solution of any problem is an individual and can be represented by a set of parameters. These parameters are regarded as the genes of a chromosome. A value, generally known as a fitness value, is used to reflect the degree of "goodness" of the chromosome for the problem. Some individuals are selected out to reproduce according to certain rules. Genetic operators are applied to obtain the genes of chromosomes of the next generation. The iteration of evaluating the fitness value, selecting the parents, and applying the genetic operators goes on until converged solution is obtained.

The GA scheme we use resembles the evolutionary strategy used in the recent experiment [7] and the semiclassical model analysis [10], but the calculation of the fitness is completely different. The procedure starts with a Gaussian pulse of $10 \mathrm{fs}$ duration and peak intensity $2 \times 10^{14} \mathrm{~W} / \mathrm{cm}^{2}$ and wavelength $800 \mathrm{~nm}$, which interacts with atomic $\mathrm{H}$, as shown in Fig. 1. Following a similar procedure used in the recent experiment [7], we consider the action of a phase-only pulse shaper [19], and transform the laser pulse into the spectral domain. Thus starting from a Fourier-transform-limited Gaussian pulse laser field, 


$$
\epsilon_{0}(t)=E_{0} e^{-t^{2} / \tau^{2}} e^{i \omega_{0} t}
$$

where $E_{0}$ is the peak field amplitude, $\omega_{0}$ is the angular frequency of $800 \mathrm{~nm}$ laser, and $\tau$ is related to the pulse duration. The field is transformed into spectral domain through Fourier transform, and the resulting pulse spectrum $E(\omega)$ is discretized into $n$ equal spacing (in $\omega$ ) sample points $\left\{E\left(\omega_{j}\right)\right\}, j=1,2, \ldots, n$, centered around $\omega_{0}$, called the "control knobs." By adjusting only the phase $\varphi_{j}$ of the laser pulse in the spectral domain, the pulse energy is conserved, but the amplitude and phase of the laser pulse in the time domain will be modified:

$$
\epsilon(t)=\frac{1}{2 \pi} \sum_{j=1}^{n} E\left(\omega_{j}\right) e^{-i \varphi_{j}} e^{i \omega_{j} t} \Delta \omega_{j}
$$

Here $\varphi_{j}, j=1, \ldots, n\left(0 \leqslant \varphi_{j} \leqslant 2 \pi\right)$, are to be optimized through the genetic algorithm. We use the following GA approach. Starting with a population $(m=20)$ of candidate pulses, whose sets of $\varphi_{j}$ 's are randomly selected, we solve the time-dependent Schrödinger equations of $\mathrm{H}$ atom in these laser fields. Using the HHG power spectrum at the chosen harmonic and the nearby harmonics as the criteria of fitness, a fraction of the population, namely, the $m_{f}$ fittest individuals, is selected as parents. Since our purpose is to enhance $\mathrm{H} 27$ intensity and simultaneously suppress the enhancement of $\mathrm{H} 25$ and $\mathrm{H} 29$ intensities, we define the fitness function as

$$
f=\sum a_{j}-\sum a_{l}-\sum a_{k}
$$

where $j, l, k$ label the pixels within $0.5 \omega_{0}$ centered around the 25th, 27th, and 29th harmonic, respectively, and $a_{i}$ $=\left|d\left(\omega_{i}\right)\right|^{2}$ is the HHG power spectrum intensity at frequency $\omega_{i} \cdot m_{f}(=4)$ individuals with the best fitness values are selected as parents. The reproducing is performed as follows: each parent pulse produces several children pulses, whose sets of $\varphi_{j}$ 's are slightly modified compared to their parent by nonuniform mutation procedure [20] with maximum number of iterations set to be 50. A new generation of candidate pulses are thus formed. The process of evaluating the fitness of each individual, selecting the parents, and performing the mutation repeats, until converged $(|\Delta f|$ $<0.001$ ) and optimized value of the fitness function is achieved. The magnitude of mutation decreases with increasing iteration number to facilitate fine tune and convergence. Typically 30-40 genetic iterations are sufficient to achieve the convergence.

The comparison of the optimized and the original HHG power spectra is shown in Fig. 3(a). We see that after the optimization, the $\mathrm{H} 27$ peak is more than one order of magnitude enhanced, and in addition, its spectral purity is markedly improved. Figure 3(b) shows the comparison of the laser-pulse shape in the time domain before and after GA optimization. Also shown is the phase values of the optimized laser fields. (The initial laser phases before optimization are taken to be zero for the whole Gaussian pulse.) We see that the optimized laser shape has a slightly smaller peak amplitude and asymmetric shape and its peak field position is
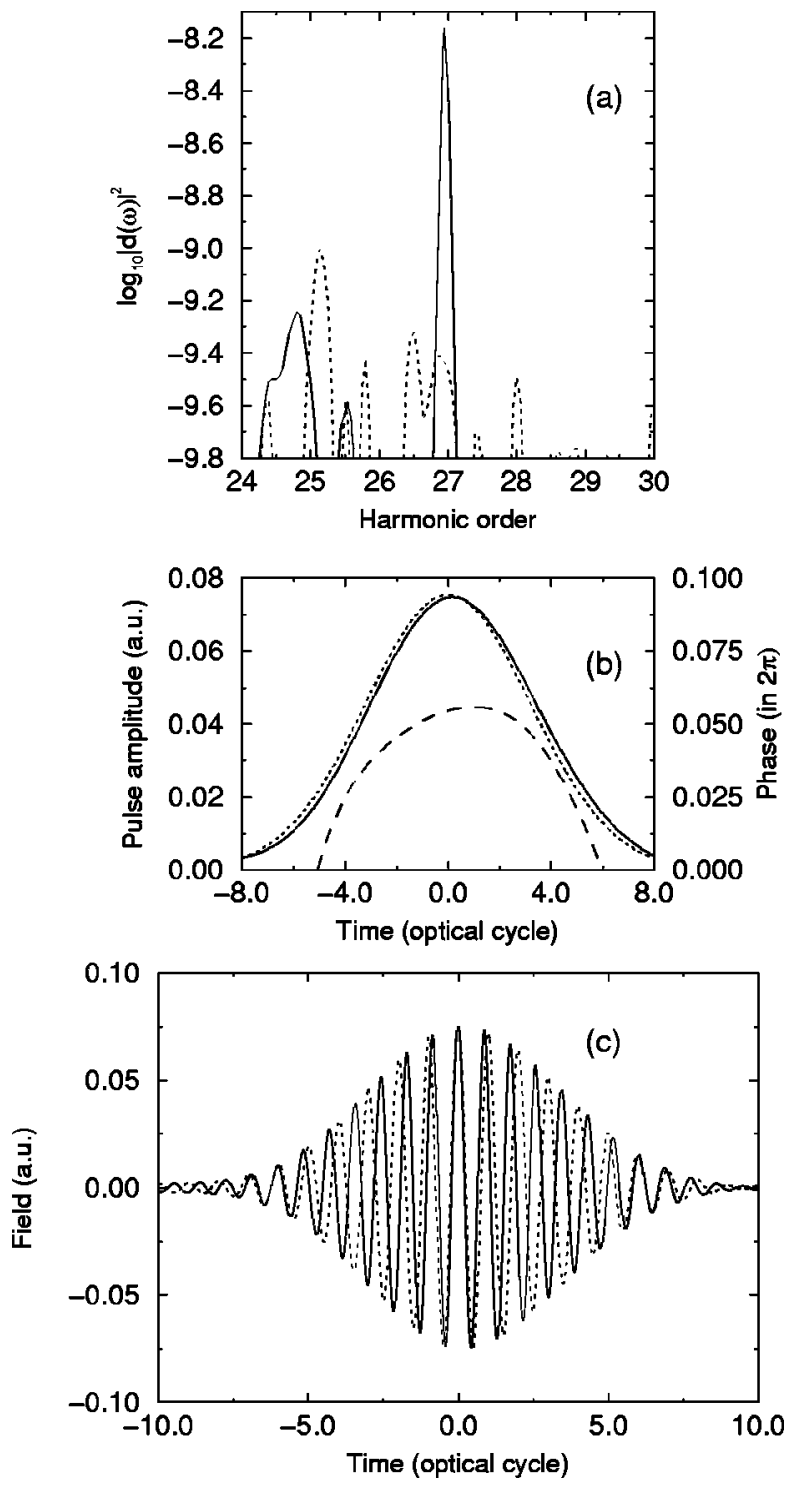

FIG. 3. (a) Optimization of the 27th harmonic in atomic H. The harmonic peak is enhanced by over an order of magnitude. Harmonic power spectra before and after optimization are shown by dotted and solid lines, respectively. (b) Comparison of the laserpulse shape before (dotted line) and after (solid line) optimization. Also shown is the phase of the optimized pulse (dashed line). (c) Time-dependent electric field before (dotted line) and after (solid line) optimization.

slightly shifted from the initial central location. The optimized pulse also has relatively longer tails in both ends with some small structure (not shown). The corresponding phase values show larger modification, similar to the behavior seen in the experimental optimization [7]. However, as shown in Fig. 2, the major time domain contributing to the total harmonic intensity is the central part of the laser pulse with significant field intensity (for example, between -4 to +4 optical cycles). Beyond this central time regime, the harmonic emission intensity is negligibly small. Thus, a small modification of the laser-pulse shape and the corresponding phases in the central pulse regime can lead to rather dramatic changes and improvement of the harmonic radiation gener- 


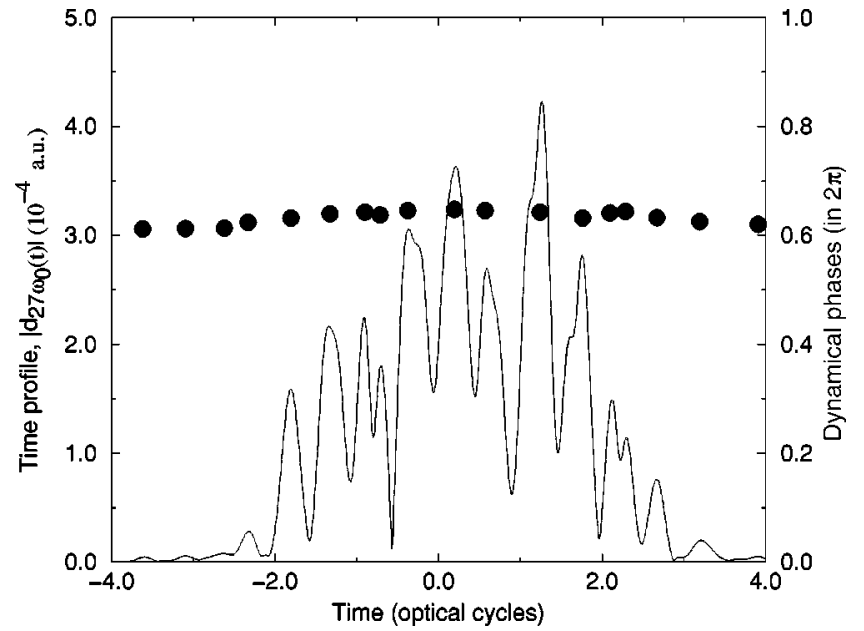

FIG. 4. The dipole-emission time profile of the 27th harmonic after optimization. The dynamical phases corresponding to those "peak" emission times $t_{q}$ are shown as black dots.

ated. Figure 3(c) shows comparison of the time-dependent electric field before and after the optimization.

Figure 4 shows the dipole-emission time profile and the corresponding dynamical phases of the 27th harmonic after the optimization. We see that the dynamical phases are now nearly uniform for all the peak emission times $t_{q}$, as opposed to the rather random behavior shown in Fig. 2 for the case before optimization. Thus, the modified laser-pulse field shown in Figs. 3(b) and 3(c) allows nearly perfect constructive interference in time of all the bremsstrahlung radiation emitted from the returning electronic wave packets within the driving laser-pulse duration, leading to significant improvement of both $\mathrm{H} 27$ peak intensity and the harmonic purity as seen in Fig. 3(a). In contrast, for the neighboring harmonics $\mathrm{H} 25$ and $\mathrm{H} 29$, our calculations show that their dynamical phases remain to be randomly distributed.

In conclusion, we have presented a fully $a b$ initio quantum study of the coherent control of the HHG processes by means of both genetic algorithm and wavelet transform techniques. It is shown that the dynamical phases of the dipoleemission time profiles play a key role in the optimization process. The use of wavelet transform and accurate solution of the time-dependent Schrödinger equation allow the determination of such important quantum dynamical phase information, taking into account the effect of atomic structure. By optimizing this intra-atomic phase matching and the laserpulse shape on the suboptical cycle time scale through the genetic algorithm, both the intensity and purity of a specific harmonic can be significantly improved. Extension of the procedure to the study of coherent control of more complex atoms and molecules is in progress.

This work was partially supported by the U.S. Department of Energy, Office of Science, Office of Basic Energy Sciences, Division of Chemical Sciences. We are grateful to the Kansas Center for Advanced Scientific Computing for the support of Origin2400 supercomputer time.
[1] H. Rabitz, R. de Vivie-Riedle, M. Motzkus, and K. Kompa, Science 288, 824 (2000).

[2] T. C. Weinacht, J. Ahn, and P. H. Bucksbaum, Nature (London) 397, 233 (1999).

[3] D. Meshulach and Y. Silberberg, Nature (London) 396, 239 (1998).

[4] R. N. Zare, Science 279, 1875 (1998).

[5] Z. Chang, A. Rundquist, H. Wang, M. M. Murnane, and H. C. Kapteyn, Phys. Rev. Lett. 79, 2967 (1997).

[6] C. Spielmann, et al., Science 278, 661 (1997).

[7] R. Bartels, S. Backus, E. Zeek, L. Mlsogutl, G. Vdovin, I. P. Christov, M. M. Murnane, and H. C. Kapteyn, Nature (London) 406, 164 (2000).

[8] M. Lewenstein, P. Balcou, M. Y. Ivanov, and P. B. Corkum, Phys. Rev. A 49, 2117 (1993).

[9] K. C. Kulander, K. J. Schafer, and J. L. Krause, in SuperIntense Laser-Atom Physics, Vol. 316 of NATO Advanced Study Institute Series B: Physics, edited by P. B. Piraux et al.
(Plenum, New York, 1993).

[10] I. P. Christov, R. Bartels, H. C. Kapteyn, and M. M. Murnane (unpublished).

[11] Evolutionary Algorithms, edited by L. D. Davis, K. De Jong, M. D. Vose, and L. D. Whitley (Springer, New York, 1999).

[12] X. M. Tong and S. I. Chu, Chem. Phys. 217, 119 (1997).

[13] G. Yao and S. I. Chu, Chem. Phys. Lett. 204, 381 (1993).

[14] X. M. Tong and S. I. Chu, Phys. Rev. A 61, 021802(R) (2000).

[15] X. M. Tong and S. I. Chu, Phys. Rev. A 57, 452 (1998); 64, 013417 (2001).

[16] X. Chu and S. I. Chu, Phys. Rev. A 63, 023411 (2001).

[17] X. M. Tong and S. I. Chu, Phys. Rev. A 61, 031401(R) (2000).

[18] C. K. Chui, An Introduction to Wavelets (Academic Press, New York, 1992).

[19] E. Zeek et al., Opt. Lett. 24, 493 (1999).

[20] L. Davis, Handbook of Genetic Algorithms (Van Nostrand Reinhold, New York, 1991). 